Bull. Korean Math. Soc. 52 (2015), No. 1, pp. 69-83

http://dx.doi.org/10.4134/BKMS.2015.52.1.069

\title{
THE SEPARABLE WEAK BOUNDED APPROXIMATION PROPERTY
}

\author{
Keun Young Lee
}

\begin{abstract}
AвSTRACT. In this paper we introduce and study the separable weak bounded approximation properties which is strictly stronger than the approximation property and but weaker than the bounded approximation property. It provides new sufficient conditions for the metric approximation property for a dual Banach space.
\end{abstract}

\section{Introduction}

Let $X$ and $Y$ be Banach spaces. We denote by $\mathcal{B}(X, Y)$ the space of bounded linear operators from $X$ into $Y$, and by $\mathcal{F}(X, Y), \mathcal{K}(X, Y), \mathcal{W}(X, Y)$, and $\mathcal{B}_{S}(X, Y)$ its subspaces of finite rank operators, compact operators, weakly compact operators, and separable-valued bounded linear operators.

Recall that a Banach space $X$ is said to have the approximation property (AP) if there exists a net $\left(S_{\alpha}\right) \subset \mathcal{F}(X, Y)$ such that $S_{\alpha} \rightarrow I_{X}$ uniformly on compact subsets of $X$. If $\left(S_{\alpha}\right)$ can be chosen with $\sup _{\alpha}\left\|S_{\alpha}\right\| \leq 1$, then $X$ is said to have the metric approximation property (MAP). The following is a long standing open problem [1].

The Metric Approximation Problem. Does the approximation property of the dual space $X^{*}$ of a Banach space $X$ imply the metric approximation property of $X^{*}$ ?

Thanks to Grothendieck, he provides an affirmative answer to the above problem in the case that $X^{*}$ is separable. Many people in this field have consider the problem, but not much progress has been obtained (see $[3,8,9$, 12]). The following theorem is the most far-reaching result on this problem.

Theorem 1 ([13, Theorem 5.50]). Let $X$ be a Banach space such that $X^{*}$ or $X^{* *}$ has the Radon-Nikodym property. If $X^{*}$ has the approximation property, then $X^{*}$ has the metric approximation property.

Received August 18, 2012; Revised July 10, 2013.

2010 Mathematics Subject Classification. Primary 46B28; Secondary 46B22.

Key words and phrases. the separable weak metric approximation property, separable weak metric approximation property with conjugate operators, weak Radon-Nikodým property, metric approximation property, factorization lemma. 
We can have the following natural question from Theorem 1.

Question. What are other non-trivial sufficient conditions which guarantee the metric approximation property for a dual Banach space $X^{*}$ ?

Our aim in this paper is to provide answers to the above question. To do this, we introduce the separable weak metric approximation property and study first a more general concept so called the separable weak $\lambda$-bounded approximation property. The separable weak metric approximation property is strictly stronger than the AP and weaker than the MAP. We need the weak RadonNikodým property. Recall that the weak Radon-Nikodým property is a strictly weaker property than the Radon-Nikodým property (see [10]).

Our paper is organized as follows. In Section 2, we fix notations and introduce basic facts. In Section 3, we study the separable weak $\lambda$-bounded approximation property. Then we provide characterizations of the separable weak $\lambda$-bounded approximation property and several properties about this. In Section 4, we give answers to Question.

\section{Preliminaries}

A Banach $X$ is said to have the Radon-Nikodym property if for each finite measure space $(\Omega, \Sigma, \mu)$ and each $\mu$-continuous $X$-valued countably additive vector measure $F: \Sigma \rightarrow X$ of bounded variation, there exists a Bochner integrable function $f: \Omega \rightarrow X$ such that $F(E)=\int_{E} f d \mu$ for all $E \in \Sigma$ (see [13]). A Banach space $X$ is said to have the weak Radon-Nikodym property if for each finite complete measure space $(\Omega, \Sigma, \mu)$ and each $\mu$-continuous $X$ valued countably additive vector measure $F: \Sigma \rightarrow X$ of bounded variation, there exists a Pettis integrable function $f: \Omega \rightarrow X$ such that $F(E)=(P)$ $\int_{E} f d \mu$ for all $E \in \Sigma$ (see [11]). Clearly if $X$ has the Radon-Nikodým property, then $X$ has the weak Radon-Nikodým property but the converse is not true. For example, $J T^{*}$ has the weak Radon-Nikodým property but does not have Radon-Nikodým property where $J T$ is the James tree space (see [10]).

Let us fix some notations. We denote by $\mathcal{B}_{w^{*}}\left(X^{*}, Y^{*}\right)\left(\mathcal{B}_{w^{*} w}\left(X^{*}, Y\right)\right)$ its subspace of weak* to weak* (weak) continuous operators. Similarly, we define $\mathcal{K}_{w^{*}}\left(X^{*}, Y^{*}\right)\left(\mathcal{K}_{w^{*} w}\left(X^{*}, Y\right)\right)$ and $\mathcal{F}_{w^{*}}\left(X^{*}, Y^{*}\right)$. Furthermore, we denote by $\mathcal{B}(X, Y ; \lambda)$ the space of bounded linear operators $T$ from $X$ to $Y$ satisfying $\|T\| \leq \lambda$. Similarly, we define $\mathcal{K}(X, Y ; \lambda)$ and $\mathcal{F}(X, Y ; \lambda)$. We denote $S_{\alpha} \stackrel{\tau}{\longrightarrow} T$ in $\mathcal{B}(X, Y)$ by $S_{\alpha} \rightarrow T$ uniformly on compact subsets of $X$.

\section{The separable weak bounded approximation property}

Let $X$ be a Banach space and let $1 \leq \lambda<\infty$. Let us recall that $X$ is said to have the $\lambda$-bounded approximation property $(\lambda$-BAP) if there exists a net $\left(S_{\alpha}\right) \subset \mathcal{F}(X, X)$ such that $\sup _{\alpha}\left\|S_{\alpha}\right\| \leq \lambda$ and $S_{\alpha} \stackrel{\tau}{\longrightarrow} I_{X}$.

Definition 3.1. A Banach space $X$ is said to have the separable weak $\lambda$ bounded approximation property (s-weak $\lambda$-BAP) if for every separable Banach 
space $Y$ and $T \in \mathcal{B}(Y, X ; 1)$,

$$
I_{X} \in \overline{\{S \in \mathcal{F}(X, X):\|S T\| \leq \lambda\}}^{\tau} .
$$

If $\lambda=1$, then we say that $X$ has the separable weak metric approximation property. Also we say that $X$ has the separable weak bounded approximation property (s-weak-BAP) if $X$ has the s-weak $\lambda$-BAP for some $\lambda$.

Let us start with following simple observations. Given two properties $P_{1}$ and $P_{2}, P_{1} \geq P_{2}$ means that $P_{1}$ is stronger than $P_{2}$.

Proposition 3.2. We have $\lambda-B A P \geq s$-weak $\lambda$-BAP. Furthermore, we obtain that s-weak BAP is strictly stronger than $A P$.

Proof. First we show that $\lambda$-BAP $\geq$ s-weak $\lambda$-BAP. Suppose that $X$ has the $\lambda$-BAP. Let $Y$ be a separable Banach space and $T \in \mathcal{B}(Y, X ; 1)$. Then there exists a net $\left(S_{\alpha}\right) \subset \mathcal{F}(X, X ; \lambda)$ such that $S_{\alpha} \stackrel{\tau}{\longrightarrow} I_{X}$. Then we have $\left\|S_{\alpha} T\right\| \leq \lambda$, hence $X$ has the s-weak $\lambda$-BAP.

For s-weak BAP $\geq \mathrm{AP}$, it is clear. Finally, let $X$ be the Figiel and Johnson space (see [4]). Then $X$ is a separable Banach space and has the AP, but it does not have the BAP. Hence we have

$$
I_{X} \notin \overline{\mathcal{F}(X, X ; \lambda)}^{\tau}
$$

for all $\lambda \geq 1$. Since $X$ is separable, $X$ does not have the s-weak BAP.

Remark 3.3. We do not know whether the s-weak BAP and the BAP are different properties. We conjecture that they are.

We now are going to investigate the characterization of the s-weak $\lambda$-BAP. To obtain this characterization, we need following lemmas. Recall that $X_{K}$ is the Davis-Figiel-Johnson-Pelczynski construction where $K$ is a closed absolutely convex subset of $B_{X}$ (see [8]).

Lemma 3.4. Suppose that $(V, \tau)$ is a locally convex space and $x \in V$. If $C$ is a balanced convex subset of $V$, then $x \in \bar{C}^{\tau}$ if and only if for every $f \in(V, \tau)^{*}$

$$
|f(x)| \leq \sup _{y \in C}|f(y)| .
$$

Proof. By continuity the "only if" part is clear. To show the "if" part, suppose to the contrary that $x \notin \bar{C}^{\tau}$. Then, by the separation theorem, there exist $f \in(V, \tau)^{*}$ and $t \in \mathbb{R}$ such that $\operatorname{Re} f(x)>t>\operatorname{Re} f(y)$ for all $y \in C$. Since $C$ is balanced,

$$
|f(x)|>t \geq \sup _{y \in C} \operatorname{Re} f(y)=\sup _{y \in C}|f(y)| .
$$

This completes the proof.

Lemma 3.5. Let $K$ be a closed absolutely convex subset of $B_{X}$ and $Z$ be $X_{K}$. Then for every Banach space $Y$

$$
\mathcal{F}(Z, Y) \subset \overline{\{S J: S \in \mathcal{F}(X, Y)\}},
$$


where $J: Z \rightarrow X$ is the identity embedding.

Proof. Let $T=\sum_{k=1}^{n} \varphi_{k}(\cdot) y_{k} \in \mathcal{F}(Z, Y)$ and $\varepsilon>0$. We may assume that $\sum_{k=1}^{n}\left\|y_{k}\right\|=1$. Since $J^{* *}: Z^{* *} \rightarrow X^{* *}$ is injective, we have $\overline{J^{*}\left(X^{*}\right)}=Z^{*}$. Then there exist $x_{1}^{*}, \ldots, x_{n}^{*} \in X^{*}$ so that

$$
\left\|\varphi_{k}-J^{*}\left(x_{k}^{*}\right)\right\|<\varepsilon
$$

for $k=1, \ldots, n$. Consider $S=\sum_{k=1}^{n} x_{k}^{*}(\cdot) y_{k} \in \mathcal{F}(X, Y)$. Then we obtain

$\|T-S J\|=\left\|\sum_{k=1}^{n} \varphi_{k}(\cdot) y_{k}-\sum_{k=1}^{n} x_{k}^{*} J(\cdot) y_{k}\right\|=\left\|\sum_{k=1}^{n} \varphi_{k}(\cdot) y_{k}-\sum_{k=1}^{n} J^{*} x_{k}^{*}(\cdot) y_{k}\right\|<\varepsilon$.

Hence $T \in \overline{\{S J: S \in \mathcal{F}(X, Y)\}}$.

Lemma 3.6 ([8, Theorem 2.2]). If $T: Y \rightarrow X$ is separably valued, then there exists a separable Banach space $Z=X_{K}, R: Y \rightarrow Z$, and $J: Z \rightarrow X$ so that $T=J R, R y=T y$ for every $y \in Y, J$ is the inclusion map, and $\|T\|=\|R\|$, $\|J\|=1$ where $K$ is $\overline{T\left(B_{Y}(0,1 /\|T\|)\right)}$.

Theorem 3.7. Let $X$ be a Banach space and let $1 \leq \lambda<\infty$. The following are equivalent.

(a) $X$ has the s-weak $\lambda$-BAP.

(b) For every separable Banach space $Y$, we have

$$
\mathcal{B}(Y, X ; 1) \subset \overline{\mathcal{F}(Y, X ; \lambda)}^{\tau} .
$$

(c) For every Banach space $Y$ and $T \in \mathcal{B}_{S}(Y, X ; 1)$, we have

$$
T \in \overline{\{S T: S \in \mathcal{F}(X, X):\|S T\| \leq \lambda\}^{\tau}} \text {. }
$$

(d) For every Banach space $Y$ and $T \in \mathcal{B}_{S}(Y, X ; 1)$, we have

$$
I_{X} \in \overline{\{S \in \mathcal{F}(X, X):\|S T\| \leq \lambda\}}{ }^{\tau} .
$$

Proof. (a) $\Rightarrow(\mathrm{b})$ and $(\mathrm{d}) \Rightarrow(\mathrm{a})$ are clear.

(b) $\Rightarrow$ (c) Let $Y$ be a Banach space and let $T \in \mathcal{B}_{S}(Y, X ; 1)$. By Lemma 3.6, there exist a separable Banach space $Z, R: Y \rightarrow Z$, and $J: Z \rightarrow X$ such that $T=J R, R y=T y$ for every $y \in Y, J$ is the inclusion mapping, $\|T\|=\|R\|$, and $\|J\|=1$. By the assumption, we have

$$
J \in \overline{\mathcal{F}(Z, X ; \lambda)}^{\tau} \text {. }
$$

By Lemma 3.5, we have

$$
\mathcal{F}(Z, X ; \lambda) \subseteq \overline{\{S J: S \in \mathcal{F}(Z, X) ;\|S J\| \leq \lambda\}},
$$

hence we obtain

$$
J \in \overline{\mathcal{F}(Z, X ; \lambda)}^{\tau}=\overline{\{S J: S \in \mathcal{F}(X, X) ;\|S J\| \leq \lambda\}}^{\tau} .
$$


Then there exists a net $\left(S_{\alpha}\right)$ in $\mathcal{F}(X, X)$ such that $\sup _{\alpha}\left\|S_{\alpha} J\right\| \leq \lambda$ and $S_{\alpha} J \stackrel{\tau}{\longrightarrow} J$. For every compact $K \subset Y$

$$
\sup _{y \in K}\left\|S_{\alpha} T y-T y\right\|=\sup _{y \in K}\left\|S_{\alpha} J R y-J R y\right\| \rightarrow 0 .
$$

Further, for every $\alpha$

$$
\left\|S_{\alpha} T\right\|=\sup _{y \in K}\left\|S_{\alpha} J R y\right\| \leq\left\|S_{\alpha} J\right\| \sup _{y \in B_{Y}}\|R y\| \leq \lambda\|R\|=\lambda\|T\| \leq \lambda .
$$

Hence we have

$$
T \in \overline{\{S T: S \in \mathcal{F}(X, X),\|S T\| \leq \lambda\}}^{\tau} .
$$

(c) $\Rightarrow$ (d) Let $Y$ be a Banach space and $T$ be in $\mathcal{B}_{S}(Y, X ; 1)$. Let $\left(x_{n}\right)$ and $\left(x_{n}^{*}\right)$ be sequences in $X$ and $X^{*}$, respectively, so that $\sum_{n=1}^{\infty}\left\|x_{n}^{*}\right\|\left\|x_{n}\right\|<\infty$. We may assume that for every $n\left\|x_{n}\right\| \leq 1, x_{n} \rightarrow 0$ and $\sum_{n=1}^{\infty=1}\left\|x_{n}^{*}\right\|<\infty$. Now let

$$
K=\overline{\operatorname{abconv}\left\{\left(x_{n}\right)_{n=1}^{\infty} \cup T\left(B_{Y}\right)\right\}} .
$$

Since $K$ is a separable subset of $X$, by the factorization lemma [8], there exists a Banach space $Z$ such that the identity embedding $J \in \mathcal{B}_{S}(Z, X),\|J\| \leq 1$, and $K \subset J\left(B_{Z}\right)$. By the assumption (c), we have

$$
J \in \overline{\{S J: S \in \mathcal{F}(X, X),\|S J\| \leq \lambda\}}^{\tau} \subset \mathcal{B}(Z, X) .
$$

Since $\sum_{n} x_{n}^{*}(\cdot) x_{n} \in(\mathcal{B}(Z, X), \tau)^{*}$, by (3.1) and Lemma 3.4, we obtain

$$
\begin{aligned}
\left|\sum_{n=1}^{\infty} x_{n}^{*}\left(x_{n}\right)\right| & =\left|\sum_{n=1} x_{n}^{*} J x_{n}\right| \\
& \leq \sup \left\{\left|\sum_{n} x_{n}^{*}\left(S J x_{n}\right)\right|: S \in \mathcal{F}(X, X),\|S J\| \leq \lambda\right\} \\
& =\sup \left\{\left|\sum_{n} x_{n}^{*}\left(S x_{n}\right)\right|: S \in \mathcal{F}(X, X),\|S J\| \leq \lambda\right\} \\
& \leq \sup \left\{\left|\sum_{n} x_{n}^{*}\left(S x_{n}\right)\right|: S \in \mathcal{F}(X, X),\|S T\| \leq \lambda\right\}
\end{aligned}
$$

because $\|S T\| \leq\|S J\|$. Since $\{S T: S \in \mathcal{F}(X, X),\|S T\| \leq \lambda\}$ is a balanced convex subset of $\mathcal{B}(Z, X)$, Lemma 3.4 implies

$$
I_{X} \in \overline{\{S \in \mathcal{F}(X, X):\|S T\| \leq \lambda\}}{ }^{\tau} .
$$

Using Theorem 3.7, we have the following corollary.

Corollary 3.8. Let $X$ be a Banach space and let $1 \leq \lambda<\infty$. The following are equivalent.

(a) $X$ has the s-weak $\lambda$-BAP. 
(b) For every Banach space $Y$, for every operator $T \in \mathcal{B}_{S}(Y, X)$ with $\|T\|=$ 1 , and for all sequence $\left(x_{n}^{*}\right) \subset X$, and $\left(y_{n}\right) \subset Y$ with $\sum_{n=1}^{\infty}\left\|x_{n}^{*}\right\|\left\|y_{n}\right\|<\infty$, one has the inequality

$$
\left|\sum_{n=1}^{\infty} x_{n}^{*} T\left(y_{n}\right)\right| \leq \lambda \sup _{\|S T\| \leq 1, S \in \mathcal{F}(X, X)}\left|\sum_{n=1}^{\infty} x_{n}^{*} S T\left(y_{n}\right)\right| .
$$

Proof. (a) $\Rightarrow$ (b) Let $Y$ be a Banach space and $T$ be in $\mathcal{B}_{S}(Y, X)$ with $\|T\|=1$. Take $\left(x_{n}^{*}\right) \subset X^{*}$, and $\left(y_{n}\right) \subset Y$ with $\sum_{n=1}^{\infty}\left\|x_{n}^{*}\right\|\left\|y_{n}\right\|<\infty$ and let $\varepsilon>0$. We choose $N \in \mathbb{N}$ such that $\sum_{n>N}\left\|x_{n}^{*}\right\|\left\|y_{n}\right\|<\varepsilon$. By the assumption and Theorem 3.7, we can choose $S \in \mathcal{F}(X, X)$ such that $\|S T\| \leq \lambda$ and $\left\|x_{n}^{*}\right\| \| S T\left(y_{n}\right)-$ $T\left(y_{n}\right) \|<\varepsilon / N$ for all $n=1,2, \ldots, N$. We obtain

$$
\begin{aligned}
\left|\sum_{n=1}^{\infty} x_{n}^{*} T\left(y_{n}\right)\right| & \leq\left|\sum_{n=1}^{N} x_{n}^{*} S T\left(y_{n}\right)\right|+\left|\sum_{n=1}^{N} x_{n}^{*} S\left(y_{n}\right)-x_{n}^{*} T\left(y_{n}\right)\right|+\sum_{n>N}\left\|x_{n}^{*}\right\|\left\|y_{n}\right\| \\
& \leq\left|\sum_{n=1}^{\infty} x_{n}^{*} S T\left(y_{n}\right)\right|+2 \varepsilon .
\end{aligned}
$$

Hence we have

$$
\begin{aligned}
\left|\sum_{n=1}^{\infty} x_{n}^{*} T\left(y_{n}\right)\right| & \leq \sup _{\|S T\| \leq \lambda, S \in \mathcal{F}(X, X)}\left|\sum_{n=1}^{\infty} x_{n}^{*} S T\left(y_{n}\right)\right| \\
& =\lambda \sup _{\|S T\| \leq 1, S \in \mathcal{F}(X, X)}\left|\sum_{n=1}^{\infty} x_{n}^{*} S T\left(y_{n}\right)\right| .
\end{aligned}
$$

(b) $\Rightarrow$ (a) Let $Y$ be a Banach space and $T$ be in $\mathcal{B}_{S}(Y, X)$ with $\|T\|=1$. By Theorem 3.7, we are enough to show

$$
T \in \overline{\{S T: S \in \mathcal{F}(X, X),\|S T\| \leq \lambda\}}^{\tau} .
$$

Take $\left(x_{n}^{*}\right) \subset X^{*}$, and $\left(y_{n}\right) \subset Y$ with $\sum_{n=1}^{\infty}\left\|x_{n}^{*}\right\|\left\|y_{n}\right\|<\infty$. By the assumption (b), we have

$$
\left|\sum_{n=1}^{\infty} x_{n}^{*} T\left(y_{n}\right)\right| \leq \sup _{\|S T\| \leq \lambda, S \in \mathcal{F}(X, X)}\left|\sum_{n=1}^{\infty} x_{n}^{*} S T\left(y_{n}\right)\right| .
$$

Since $\sum_{n} x_{n}^{*}(\cdot) y_{n} \in(\mathcal{B}(Y, X), \tau)^{*}$ and $\{S T: S \in \mathcal{F}(X, X),\|S T\| \leq \lambda\}$ is a balanced convex subset of $\mathcal{B}(Y, X)$, by Lemma 3.4, we obtain

$$
T \in \overline{\{S T: S \in \mathcal{F}(X, X),\|S T\| \leq \lambda\}}^{\tau} .
$$

This completes the proof.

Let us consider the trace mapping $V$ from the projective tensor product $X^{*} \hat{\otimes}_{\pi} Y$ to $\mathcal{F}(Y, X)^{*}$ defined by

$$
V(u)(T)=\operatorname{trace}(T u), u \in X^{*} \hat{\otimes}_{\pi} Y, T \in \mathcal{F}(Y, X) .
$$


This means that if $u=\sum_{n} x_{n}^{*} \otimes y_{n}$ with $\sum_{n}\left\|x_{n}^{*}\right\|\left\|y_{n}\right\|<\infty$, then

$$
(V u)(T)=\sum_{n} x_{n}^{*}\left(T\left(y_{n}\right)\right)
$$

It is well known that

$$
\|V u\| \leq\|u\|_{\pi}, u \in X^{*} \hat{\otimes}_{\pi} Y
$$

Applying the trace mapping, Grothendieck [5] proved that a Banach space $X^{*}$ has the $\lambda$-BAP if and only if, for every Banach space $Y$, the trace mapping $V: X^{*} \hat{\otimes}_{\pi} Y \rightarrow \mathcal{F}(Y, X)^{*}$ satisfies $\|u\|_{\pi} \leq \lambda\|V u\|$ for all $u \in X^{*} \hat{\otimes}_{\pi} Y$. On the other hand, Lima and Oja [9] showed that a Banach space $X^{*}$ has the AP if and only if, for every separable reflexive Banach space $Y$, the trace mapping $V: X^{*} \hat{\otimes}_{\pi} Y \rightarrow \mathcal{F}(Y, X)^{*}$ satisfies $\|u\|_{\pi} \leq \lambda\|V u\|$ for all $u \in X^{*} \hat{\otimes}_{\pi} Y$. The following theorem provides some relation between the s-weak $\lambda$-BAP and the trace mapping.

Theorem 3.9. Suppose that for every separable Banach space $Y$, the trace mapping $V: X^{*} \hat{\otimes}_{\pi} Y \rightarrow \mathcal{F}(Y, X)^{*}$ satisfies $\|u\|_{\pi} \leq \lambda\|V u\|$ for all $u \in X^{*} \hat{\otimes}_{\pi} Y$. Then $X$ has the s-weak $\lambda$-BAP.

Proof. We are enough to verify condition (b) of Corollary 3.8. Let $Y$ be a Banach space and $T$ be in $\mathcal{B}_{S}(Y, X)$ with $\|T\|=1$. Take $\left(x_{n}^{*}\right) \subset X^{*}$, and $\left(y_{n}\right) \subset Y$ with $\sum_{n=1}^{\infty}\left\|x_{n}^{*}\right\|\left\|y_{n}\right\|<\infty$. We may assume that $\left\|y_{n}\right\|=1, n \in \mathbb{N}$. Let $K=\overline{T\left(B_{Y}\right)}$. By Lemma 3.6, there exists a separable Banach space $Z=X_{K}$ so that $J: Z \rightarrow X$ is the inclusion mapping and $K \subset J\left(B_{Z}\right)$. Let $z_{n} \in B_{Z}$ be such that $T\left(y_{n}\right)=J\left(z_{n}\right), n \in \mathbb{N}$. Put

$$
u=\sum_{n} x_{n}^{*} \otimes z_{n} \in X^{*} \hat{\otimes}_{\pi} Z .
$$

Consider the trace mapping $V: X^{*} \hat{\otimes}_{\pi} Z \rightarrow \mathcal{F}(Z, X)^{*}$. Since $Z$ is separable, by the assumption, we have $\|u\|_{\pi} \leq \lambda\|V u\|$. By Lemma 3.5, we obtain that

$$
\mathcal{F}(Z, X ; 1) \subset \overline{\{S J: S \in \mathcal{F}(X, X),\|S J\| \leq 1\}} .
$$

Note that $\|S T\| \leq\|S J\|$ for all $S \in \mathcal{F}(X, X)$ because $T\left(B_{Y}\right) \subseteq J\left(B_{Z}\right)$. Then we have

$$
\begin{aligned}
\left|\sum_{n} x_{n}^{*} T\left(y_{n}\right)\right| & =\left|\sum_{n=1}^{\infty} x_{n}^{*} J\left(z_{n}\right)\right|=|\operatorname{trace}(J u)| \\
& \leq\|J\|\|u\|_{\pi} \\
& \leq\|u\|_{\pi} \\
& \leq \lambda\|V(u)\|=\lambda \sup _{S \in \mathcal{F}(Z, X ; 1)}|\operatorname{trace}(S u)| \\
& \leq \lambda \sup _{\|S J\| \leq 1, S \in \mathcal{F}(X, X)}|\operatorname{trace}(S J u)| \\
& \leq \lambda \sup _{\|S T\| \leq 1, S \in \mathcal{F}(X, X)}\left|\sum_{n=1}^{\infty} x_{n}^{*} S J\left(z_{n}\right)\right|
\end{aligned}
$$




$$
\begin{aligned}
& =\lambda \sup _{\|S T\| \leq 1, S \in \mathcal{F}(X, X)}\left|\sum_{n=1}^{\infty} x_{n}^{*} S T\left(y_{n}\right)\right| \\
& \leq \lambda\|V(u)\| .
\end{aligned}
$$

Corollary 3.10. Suppose that for every separable Banach space $Y$, the trace mapping $V: X^{*} \hat{\otimes}_{\pi} Y \rightarrow \mathcal{F}(Y, X)^{*}$ is isometric. Then $X$ has the s-weak MAP.

Also we have the following proposition.

Proposition 3.11. Let $Z$ be an 1-complemented subspace of $X$. If $X$ has the $s$-weak $\lambda$-BAP, then $Z$ has the $s$-weak $\lambda$-BAP.

Proof. Let $Y$ be a separable Banach space and $j_{Z}$ be the inclusion mapping from $Z$ into $X$ and $P$ be the norm 1-projection from $X$ into $Z$ and $T$ be in $\mathcal{B}(Y, Z)$. By the assumption, there exists a net $\left(T_{\alpha}\right) \subset \mathcal{F}(X, X)$ such that $\sup _{\alpha}\left\|T_{\alpha} j_{Z} T\right\| \leq \lambda$ and $T_{\alpha} \stackrel{\tau}{\longrightarrow} I_{X}$. Put $S_{\alpha}=P T_{\alpha} j_{Z}$. Then a net $\left(S_{\alpha}\right)$ is in $\mathcal{F}(Z, Z)$ and we obtain

$$
\left\|S_{\alpha} T\right\|=\left\|P T_{\alpha} j_{Z} T\right\| \leq\left\|T_{\alpha} j_{Z} T\right\| \leq \lambda
$$

for all $\alpha$. Finally we are enough to show that $S_{\alpha} \stackrel{\tau}{\longrightarrow} I_{Z}$. Let $K$ be a compact subset of $Z$ and $\varepsilon>0$. Then there exists $\beta$ such that $\sup _{y \in K} \| T_{\alpha} j_{Z}(y)-$ $j_{Z}(y) \|<\varepsilon$ whenever $\alpha \succeq \beta$. Let $\alpha \succeq \beta$. Since $P j_{Z}(y)=y$ for all $y \in Z$, we obtain

$$
\sup _{y \in K}\left\|S_{\alpha}(y)-y\right\|=\sup _{y \in K}\left\|P T_{\alpha} j_{Z}(y)-P j_{Z}(y)\right\| \leq \sup _{y \in K}\left\|T_{\alpha} j_{Z}(y)-j_{Z}(y)\right\|<\varepsilon .
$$

This means that $S_{\alpha} \stackrel{\tau}{\longrightarrow} I_{Z}$. So we have

$$
I_{Z} \in \overline{\{S \in \mathcal{F}(Z, Z):\|S T\| \leq \lambda\}}{ }^{\tau},
$$

hence $Z$ has the s-weak $\lambda$-BAP.

Recall that $X^{*}$ is an 1-complemented subspace of $X^{* * *}$. Thus we have the following corollary.

Corollary 3.12. If $X^{* * *}$ has the $s$-weak $\lambda$-BAP, then $X^{*}$ has the $s$-weak $\lambda$ $B A P$.

It is useful to extend the notion of the s-weak $\lambda$-BAP with conjugate operators as follows.

Definition 3.13. A dual space $X^{*}$ of a Banach space $X$ has the s-weak $\lambda$ BAP with conjugate operators if for every separable Banach space $Y$ and $T \in$ $\mathcal{B}_{S}\left(Y, X^{*} ; 1\right)$,

that is,

$$
I_{X *} \in{\overline{\left\{S^{*}: S \in \mathcal{F}(X, X),\left\|S^{*} T\right\| \leq \lambda\right\}}}^{\tau},
$$

$$
I_{X^{*}} \in{\overline{\left\{S \in \mathcal{F}_{w^{*}}\left(X^{*}, X^{*}\right):\|S T\| \leq \lambda\right\}}}^{\tau} .
$$

If $\lambda=1$, then we say that $X^{*}$ has the separable weak metric approximation property with conjugate operators. 
Lemma $3.14([2])$. For every Banach space $X$,

$$
\mathcal{F}\left(X^{*}, X^{*}\right) \subset \overline{\mathcal{F}}_{w^{*}\left(X^{*}, X^{*}\right)}^{\tau} .
$$

Furthermore, for each $\lambda>0$,

$$
{\overline{\mathcal{F}\left(X^{*}, X^{*} ; \lambda\right)}}^{\tau} \subset \overline{\mathcal{F}}_{w^{*}\left(X^{*}, X^{*} ; \lambda\right)}{ }^{\tau} .
$$

Using the above lemma, we obtain the following relation.

Proposition 3.15. We have $\lambda-B A P \geq s$-weak $\lambda$-BAP with conjugate operators $\geq$ s-weak $\lambda$-BAP $\geq A P$ in dual Banach space.

Proof. For s-weak $\lambda$-BAP with conjugate operators $\geq$ s-weak $\lambda$-BAP, it is clear. We show that $\lambda$-BAP $\geq$ s-weak $\lambda$-BAP with conjugate operators. Suppose that $X^{*}$ has the $\lambda$-BAP. Let $Y$ be a separable Banach space and $T \in \mathcal{B}\left(Y, X^{*} ; 1\right)$. By the assumption and Lemma 3.14, we have

$$
I_{X^{*}} \in \overline{\mathcal{F}}_{w^{*}}\left(X^{*}, X^{*} ; \lambda\right)^{\tau} .
$$

Since $\|T\| \leq 1$, we obtain

$$
I_{X^{*}} \in \overline{\left\{S \in \mathcal{F}_{w^{*}}\left(X^{*}, X^{*}\right):\|S T\| \leq \lambda\right\}}{ }^{\tau} .
$$

Also we provide the following corollary which is similar to Corollary 3.8 .

Corollary 3.16. Let $X$ be a Banach space and let $1 \leq \lambda<\infty$. The following are equivalent.

(a) $X^{*}$ has the $s$-weak $\lambda$-BAP with conjugate operators.

(b) For every Banach space $Y$ and $T \in \mathcal{B}_{S}\left(Y, X^{*} ; 1\right)$,

$$
T \in \overline{\left\{S T: S \in \mathcal{F}_{w^{*}}\left(X^{*}, X^{*}\right),\|S T\| \leq \lambda\right\}}{ }^{\tau} .
$$

(c) For every Banach space $Y$, for every operator $T \in \mathcal{B}_{S}\left(Y, X^{*}\right)$ with $\|T\|=$ 1 , and for all sequence $\left(x_{n}^{* *}\right) \subset X$, and $\left(y_{n}\right) \subset Y$ with $\sum_{n=1}^{\infty}\left\|x_{n}^{* *}\right\|\left\|y_{n}\right\|<\infty$, one has the inequality

$$
\left|\sum_{n=1}^{\infty} x_{n}^{* *} T\left(y_{n}\right)\right| \leq \lambda \sup _{\|S T\| \leq 1, S \in \mathcal{F}_{w^{*}}\left(X^{*}, X^{*}\right)}\left|\sum_{n=1}^{\infty} x_{n}^{* *} S T\left(y_{n}\right)\right| .
$$

Proof. (a) $\Leftrightarrow(\mathrm{b})$ Since $F_{w^{*}}\left(X^{*}, X^{*}\right)$ is a balanced convex subset of $B\left(X^{*}, X^{*}\right)$, by the proof of Theorem $3.7((\mathrm{c}) \Rightarrow(\mathrm{d}))$, it is clear.

(b) $\Leftrightarrow(\mathrm{c})$ It comes from the proof of Corollary 3.8 .

Remark 3.17. We do not know whether the s-weak $\lambda$-BAP with conjugate operators, the s-weak $\lambda$-BAP and the AP are different properties in dual Banach spaces. We conjecture that they are. However, if $X^{*}$ has the Radon-Nikodým property, then these properties are same. 
Let us consider a Banach space $Y$ and take $T \in \mathcal{B}_{S}\left(Y, X^{*}\right)$. We define $\mathcal{F}_{T}\left(Y, X^{*}\right)$ by

$$
\mathcal{F}_{T}\left(Y, X^{*}\right)=\left\{S^{*} T: S \in \mathcal{F}(X, X)\right\} .
$$

Clearly, $\mathcal{F}_{T}\left(Y, X^{*}\right)$ is a subspace of $\mathcal{F}\left(Y, X^{*}\right)$. Then we consider the trace mapping $V$ from $X^{* *} \hat{\otimes}_{\pi} Y$ to $\mathcal{F}_{T}\left(Y, X^{*}\right)^{*}$, defined by

$$
V(u)\left(S^{*} T\right)=\operatorname{trace}\left(S^{*} T u\right), u \in X^{* *} \hat{\otimes}_{\pi} Y, S^{*} T \in \mathcal{F}_{T}\left(Y, X^{*}\right) .
$$

This means that if $u=\sum_{n} x_{n}^{* *} \otimes y_{n}$ with $\sum_{n}\left\|x_{n}^{* *}\right\|\left\|y_{n}\right\|<\infty$, then

$$
(V u)\left(S^{*} T\right)=\sum_{n} x_{n}^{* *}\left(S^{*} T\left(y_{n}\right)\right) .
$$

We observe that $\|V u\| \leq\|u\|$ for all $u \in X^{* *} \hat{\otimes}_{\pi} Y$. Also we define $W_{T}$ from $X^{* *} \hat{\otimes}_{\pi} Y$ to $\mathcal{B}(X, X)^{*}$ by

$$
W_{T}(u)(R)=\sum_{n} x_{n}^{* *}\left(R^{*} T\left(y_{n}\right)\right)
$$

whenever $u=\sum_{n} x_{n}^{* *} \otimes y_{n}$ with $\sum_{n}\left\|x_{n}^{* *}\right\|\left\|y_{n}\right\|<\infty$ and $R \in \mathcal{B}(X, X)$.

The following theorem provides relations between s-weak $\lambda$-BAP with conjugate operators and the trace mapping.

Theorem 3.18. Let $X$ be a Banach space and let $1 \leq \lambda<\infty$.

(a) For every Banach space $Y$ and $T \in \mathcal{B}_{S}\left(Y, X^{*} ; 1\right)$, the trace mapping

$$
V: X^{* *} \hat{\otimes}_{\pi} Y \rightarrow \mathcal{F}_{T}(Y, X)^{*}
$$

satisfies $\|u\|_{\pi} \leq \lambda\|V u\|$ for all $u \in X^{* *} \hat{\otimes}_{\pi} Y$.

(b) $X^{*}$ has the s-weak $\lambda$-BAP with conjugate operators.

(c) For every Banach space $Y$ and $T \in \mathcal{B}_{S}\left(Y, X^{*} ; 1\right)$, there exists an one-toone operator

$$
\Phi: \mathcal{F}_{T}(Y, X)^{*} \rightarrow \mathcal{B}(X, X)^{*}
$$

such that $\|\Phi\| \leq \lambda, \Phi(f)(R)=f\left(R^{*} T\right)$ for all $f \in \mathcal{F}_{T}(Y, X)^{*}$ and all $R \in$ $\mathcal{F}(X, X)$, and $\Phi \circ V=W_{T}$, where $V: X^{* *} \hat{\otimes}_{\pi} Y \rightarrow \mathcal{F}_{T}(Y, X)^{*}$ is the trace mapping.

Then we have $(\mathrm{a}) \Rightarrow(\mathrm{b}) \Rightarrow(\mathrm{c})$.

Proof. $(\mathrm{a}) \Rightarrow(\mathrm{b})$ The proof is similar to the proof of Theorem 3.9. We are enough to verify condition (c) of Corollary 3.16. Let $Y$ be a Banach space and $T$ be in $\mathcal{B}_{S}\left(Y, X^{*}\right)$ with $\|T\|=1$. Take $\left(x_{n}^{* *}\right) \subset X^{* *}$, and $\left(y_{n}\right) \subset Y$ with $\sum_{n=1}^{\infty}\left\|x_{n}^{* *}\right\|\left\|y_{n}\right\|<\infty$. We may assume that $\left\|y_{n}\right\|=1, n \in \mathbb{N}$. Let $K=\overline{T\left(B_{Y}\right)}$. As in the proof of Theorem 3.9, there exists a separable Banach space $Z=X_{K}$ so that $J: Z \rightarrow X$ is the inclusion map and $K \subset J\left(B_{Z}\right)$. Let $z_{n} \in B_{Z}$ be such that $T\left(y_{n}\right)=J\left(z_{n}\right), n \in \mathbb{N}$. Put

$$
u=\sum_{n} x_{n}^{* *} \otimes z_{n} \in X^{* *} \hat{\otimes}_{\pi} Z
$$


Consider the trace mapping $V: X^{* *} \hat{\otimes}_{\pi} Z \rightarrow \mathcal{F}_{J}(Z, X)^{*}$. Then we have $\|u\|_{\pi} \leq$ $\lambda\|V u\|$. Note that

$$
\mathcal{F}_{J}\left(Z, X^{*} ; 1\right)=\left\{S^{*} J: S \in \mathcal{F}(X, X),\left\|S^{*} J\right\| \leq 1\right\} .
$$

Then we have

$$
\begin{aligned}
\left|\sum_{n=1}^{\infty} x_{n}^{* *} T\left(y_{n}\right)\right| & =\left|\sum_{n=1}^{\infty} x_{n}^{*} J\left(z_{n}\right)\right|=|\operatorname{trace}(J u)| \\
& \leq\|J\|\|u\|_{\pi} \leq\|u\|_{\pi} \leq \lambda\|V(u)\| \\
& =\lambda \sup _{S^{*} J \in \mathcal{F}\left(Z, X^{*} ; 1\right)}\left|\operatorname{trace}\left(S^{*} J u\right)\right| \\
& \leq \lambda \sup _{\left\|S^{*} T\right\| \leq 1, S \in \mathcal{F}(X, X)}\left|\sum_{n=1}^{\infty} x_{n}^{* *} S^{*} J\left(z_{n}\right)\right| \\
& =\lambda \sup _{\left\|S^{*} T\right\| \leq 1, S \in \mathcal{F}(X, X)}\left|\sum_{n=1}^{\infty} x_{n}^{* *} S^{*} T\left(y_{n}\right)\right| .
\end{aligned}
$$

(b) $\Rightarrow$ (c) Let $Y$ be a Banach space and $T$ be in $\mathcal{B}_{S}\left(Y, X^{*} ; 1\right)$. Let us take a net $\left(S_{\alpha}\right) \subset \mathcal{F}(X, X)$ such that $S_{\alpha}^{*} \stackrel{\tau}{\longrightarrow} I_{X^{*}}$ and $\left\|S^{*} T\right\| \leq 1$. Since $\left(S_{\alpha}^{*} T\right) \subset$ $B_{\mathcal{F}_{T}\left(Y, X^{*}\right)^{* *}}$, which is weak ${ }^{*}$ compact, after passing to a subnet, we may assume that $\lim _{\alpha} f\left(S_{\alpha}^{*} T\right)$ exists for all $f \in \mathcal{F}_{T}\left(Y, X^{*}\right)^{*}$ (see [6]). For each $R \in \mathcal{B}(X, X)$ and $f \in \mathcal{F}_{T}\left(Y, X^{*}\right)^{*}$, we define $f_{R}: F_{T}\left(Y, X^{*}\right) \rightarrow \mathbb{F}$ by $f_{R}\left(S^{*} T\right)=f\left(R^{*} S^{*} T\right)$. It is well defined because $S R \in \mathcal{F}(X, X)$ for all $S \in \mathcal{F}(X, X)$. Also, it is easy to check that $f_{R} \in \mathcal{F}_{T}\left(Y, X^{*}\right)^{*}$. Then we can define $\Phi: \mathcal{F}_{T}\left(Y, X^{*}\right)^{*} \rightarrow \mathcal{B}(X, X)^{*}$ by

$$
(\Phi f)(R)=\lim _{\alpha} f_{R}\left(S_{\alpha}^{*} T\right)=\lim _{\alpha} f\left(R^{*} S_{\alpha}^{*} T\right)
$$

for each $R \in \mathcal{B}(X, X)$ and $f \in \mathcal{F}_{T}\left(Y, X^{*}\right)^{*}$ because $\lim _{\alpha} f\left(S_{\alpha}^{*} T\right)$ exists for every $f \in \mathcal{F}_{T}\left(Y, X^{*}\right)^{*}$. Since $\left\|S_{\alpha}^{*} T\right\| \leq \lambda$ for all $\alpha$, we obtain $\|\Phi\| \leq \lambda$.

Now we claim that $\Phi(f)(R)=f\left(R^{*} T\right)$ for all $f \in \mathcal{F}_{T}\left(Y, X^{*}\right)^{*}$ and all $R \in$ $\mathcal{F}(X, X)$. Let $f$ be in $\mathcal{F}_{T}\left(Y, X^{*}\right)^{*}, R$ in $\mathcal{F}(X, X)$ and $\varepsilon>0$. Since $R$ is a finite rank operator, $R$ is represented by $R=\sum_{k=1}^{n} x_{k}^{*}(\cdot) x_{k}$ where $\left(x_{k}\right) \subset X$ and $\left(x_{k}^{*}\right) \subset X^{*}$. We may assume that $\sum_{k=1}^{n}\left\|x_{k}\right\|=1$. Since $S_{\alpha}^{*} \stackrel{\tau}{\longrightarrow} I_{X^{*}}$, there exists $\beta$ such that if $\alpha \succeq \beta$, then

$$
\left\|S_{\alpha}^{*}\left(x_{k}^{*}\right)-x_{k}^{*}\right\|<\varepsilon
$$

for all $k=1,2, \ldots, n$. Let us fix $\alpha \succeq \beta$. Then we have

$$
\begin{aligned}
\left\|R^{*} S_{\alpha}^{*} T-R^{*} T\right\| & \leq\left\|R^{*} S_{\alpha}^{*}-R^{*}\right\| \\
& =\sup _{x^{*} \in B_{X^{*}}}\left\|\sum_{k=1}^{n} S_{\alpha}^{*}\left(x_{k}^{*}\right) x^{*}\left(x_{k}\right)-\sum_{k=1}^{n} x_{k}^{*} x^{*}\left(x_{k}\right)\right\| \\
& \leq \sum_{k=1}^{n}\left\|S_{\alpha}^{*}\left(x_{k}^{*}\right)-x_{k}^{*}\right\|\left\|x_{k}\right\|<\varepsilon .
\end{aligned}
$$


This completes our claim. Furthermore, we observe that $\Phi$ is an one-to-one operator.

Finally, we show that $\Phi \circ V=W_{T}$, where $V: X^{* *} \hat{\otimes}_{\pi} Y \rightarrow \mathcal{F}_{T}(Y, X)^{*}$ is the trace mapping. Let $u=x^{* *} \otimes y \in X^{* *} \hat{\otimes}_{\pi} Y$ and $R \in \mathcal{B}(X, X)$. Then we have

$$
\begin{aligned}
\Phi(V u)(R) & =\lim _{\alpha}\left(x^{* *} \otimes y\right)\left(R^{*} S_{\alpha}^{*} T\right) \\
& =\lim _{\alpha} x^{* *}\left(R^{*} S_{\alpha}^{*} T(y)\right) \\
& =x^{* *}\left(R^{*} T(y)\right)=W_{T}(u)(R) .
\end{aligned}
$$

Remark 3.19. We do not know whether (a), (b) and (c) in Theorem 3.18 are equivalent. We conjecture that they are.

\section{Answers to Question}

In this section, we give answers to Question as mentioned in Introduction. For this work, we need the following representation of the dual space of a compact operator space.

Theorem 4.1 ([7, Theorem 3.5]). Let $X$ and $Y$ be Banach spaces such that $X^{* *}$ or $Y^{*}$ has the weak Radon-Nikodym property. Then, for all $\phi \in \mathcal{K}(X, Y)^{*}$, there exist a sequence $\left(\left(\left(x_{i}^{n}\right)^{* *}\right)_{i=1}^{m_{n}}\right)_{n=1}^{\infty}$ in $X^{* *}$ and a sequence $\left(\left(\left(y_{i}^{n}\right)^{*}\right)_{i=1}^{m_{n}}\right)_{n=1}^{\infty}$ in $Y^{*}$ such that

$$
\langle\phi, T\rangle=\lim _{n \rightarrow \infty} \sum_{i=1}^{m_{n}}\left(x_{i}^{n}\right)^{* *}\left(T^{*}\left(\left(y_{i}^{n}\right)^{*}\right)\right)
$$

for all $T \in \mathcal{K}(X, Y)$, and

$$
\underset{n}{\lim \sup _{n}} \sum_{i=1}^{m_{n}}\left\|\left(x_{i}^{n}\right)^{* *}\right\|\left\|\left(y_{i}^{n}\right)^{*}\right\| \leq\|\phi\| .
$$

Theorem 4.2 ([7, Corollary 4.4]). Suppose that $X^{*}$ or $Y^{*}$ has the weak RadonNikodym property. Then, for all $\phi \in \mathcal{K}_{w^{*} w}\left(X^{*}, Y\right)^{*}$, there exist a sequence $\left(\left(\left(x_{i}^{n}\right)^{*}\right)_{i=1}^{m_{n}}\right)_{n=1}^{\infty}$ in $X^{*}$ and a sequence $\left(\left(\left(y_{i}^{n}\right)^{*}\right)_{i=1}^{m_{n}}\right)_{n=1}^{\infty}$ in $Y^{*}$ and

$$
\langle\phi, T\rangle=\lim _{n \rightarrow \infty} \sum_{i=1}^{m_{n}}\left(y_{i}^{n}\right)^{*}\left(T\left(\left(x_{i}^{n}\right)^{*}\right)\right.
$$

for all $T \in \mathcal{K}_{w^{*} w}\left(X^{*}, Y\right)$, and

$$
\limsup _{n} \sum_{i=1}^{m_{n}}\left\|\left(x_{i}^{n}\right)^{*}\right\|\left\|\left(y_{i}^{n}\right)^{*}\right\| \leq\|\phi\| .
$$

The following theorem provides one sufficient condition which guarantees the metric approximation property for a dual Banach space as mentioned. We modify the proof in $[12$, p. 326].

Theorem 4.3. Let $X$ be a Banach space and let $1 \leq \lambda<\infty$. Suppose that $X^{* *}$ has the weak Radon-Nikodym property. If $X^{*}$ has the s-weak $\lambda$-BAP, then $X^{*}$ has the $\lambda-B A P$. 
Proof. Let $I_{X^{*}}$ be the identity operator and $K$ be a compact subset of $X$ and let $\varepsilon>0$. Consider the seminorm

$$
p(A)=\sup \left\{\left\|A x^{*}\right\|: x^{*} \in K\right\}, A \in \mathcal{B}\left(X^{*}, X^{*}\right)
$$

and put

$$
C=\left\{S: S \in \mathcal{F}\left(X^{*}, X^{*}\right), p\left(S-I_{X^{*}}\right) \leq \varepsilon / 2\right\} .
$$

Since $X^{*}$ has the AP, $C$ is a nonempty convex subset of $\mathcal{F}\left(X^{*}, X^{*}\right)$. Let us fix $\delta>0$ such that

$$
\frac{\delta}{\lambda+\delta}\left(\varepsilon / 2+p\left(I_{X}\right)\right) \leq \varepsilon / 2
$$

Then we are enough to show that $C \cap(\lambda+\delta) B_{\mathcal{F}\left(X^{*}, X^{*}\right)} \neq \emptyset$. Suppose to the contrary that

$$
C \cap(1+\delta) B_{\mathcal{F}\left(X^{*}, X^{*}\right)}=\emptyset .
$$

By the separation theorem, there exists $\phi \in \mathcal{F}\left(X^{*}, X^{*}\right)^{*}$ such that $\|\phi\|=1$ and

$$
\lambda+\delta=\sup \{\operatorname{Re} \phi(A):\|A\| \leq \lambda+\delta\} \leq \inf \{\operatorname{Re} \phi(A): A \in C\} .
$$

By Theorem 5.1 and the Hahn-Banach theorem, there exist a sequence

$$
\left(\left(\left(x_{i}^{n}\right)^{*}\right)_{i=1}^{m_{n}}\right)_{n=1}^{\infty}
$$

in $X^{*}$ and a sequence $\left(\left(\left(x_{i}^{n}\right)^{* *}\right)_{i=1}^{m_{n}}\right)_{n=1}^{\infty}$ in $X^{* *}$ such that

$$
\langle\phi, T\rangle=\lim _{n \rightarrow \infty} \sum_{i=1}^{m_{n}}\left(x_{i}^{n}\right)^{* *}\left(T\left(\left(x_{i}^{n}\right)^{*}\right)\right)
$$

for all $T \in \mathcal{F}\left(X^{*}, X^{*}\right)$ and

$$
\limsup _{n} \sum_{i=1}^{m_{n}}\left\|\left(x_{i}^{n}\right)^{* *}\right\|\left\|\left(x_{i}^{n}\right)^{*}\right\| \leq\|\phi\| .
$$

Denote by $\mathcal{C}$ the closed absolutely convex hull in $X^{*}$ of the separable set $\left\{\left(\left(\frac{\left(x_{i}^{n}\right)^{*}}{\left\|\left(x_{i}^{n}\right)^{*}\right\|}\right)_{i=1}^{m_{n}}\right)_{n=1}^{\infty}\right\}$. By the factorization lemma [8], there exists a Banach space $Z$, which is a linear subspace of $X^{*}$, such that the identity embedding $J: Z \rightarrow X^{*}$ is a separable valued operator and $\|J\| \leq 1$. Also we have

$$
\left\{\left(\left(\frac{\left(x_{i}^{n}\right)^{*}}{\left\|\left(x_{i}^{n}\right)^{*}\right\|}\right)_{i=1}^{m_{n}}\right)_{n=1}^{\infty}\right\} \subset J\left(B_{Z}\right) .
$$

Since $X^{*}$ has the s-weak $\lambda$-BAP, there exists $S \in \mathcal{F}\left(X^{*}, X^{*}\right)$ such that $\|S J\| \leq$ $\lambda$ and $p\left(S-I_{X^{*}}\right) \leq \varepsilon / 2$. Since $S \in C$, we obtain

$$
\lambda+\delta \leq|\phi(S)| .
$$

Put $\left(\left(z_{i}^{n}\right)_{i=1}^{m_{n}}\right)_{n=1}^{\infty} \subset B_{Z}$ such that $\left(x_{i}^{n}\right)^{*}=\left\|\left(x_{i}^{n}\right)^{*}\right\| J\left(z_{i}^{n}\right)$ for all $i, n$. Then we have

$$
|\phi(S)|=\left|\lim _{n \rightarrow \infty} \sum_{i=1}^{m_{n}}\left(x_{i}^{n}\right)^{* *}\left(S\left(x_{i}^{n}\right)^{*}\right)\right|
$$




$$
\begin{aligned}
& =\left|\lim _{n \rightarrow \infty} \sum_{i=1}^{m_{n}}\left\|\left(x_{i}^{n}\right)^{*}\right\|\left(x_{i}^{n}\right)^{* *}\left(S J\left(z_{i}^{n}\right)\right)\right| \\
& \leq\|S J\| \limsup _{n} \sum_{i=1}^{m_{n}}\left\|\left(x_{i}^{n}\right)^{* *}\right\|\left\|\left(x_{i}^{n}\right)^{*}\right\| \\
& \leq\|\phi\|<\lambda+\delta,
\end{aligned}
$$

which is a contradiction.

We also give another sufficient condition which guarantees the metric approximation property for a dual Banach space. The proof is similar to Theorem 4.3. We just replace $\mathcal{F}\left(X^{*}, X^{*}\right)$ by $\mathcal{F}_{w^{*}}\left(X^{*}, X^{*}\right)$ and apply Theorem 4.2.

Theorem 4.4. Let $X$ be a Banach space and let $1 \leq \lambda<\infty$. Suppose that $X^{*}$ has the weak Radon-Nikodym property. If $X^{*}$ has the s-weak $\lambda$-BAP with conjugate, then $X^{*}$ has the $\lambda$-BAP.

Remark 4.5. Recall Theorem 1 in Introduction, that is, if $X^{*}$ has the AP and the Radon-Nikodým property, then $X^{*}$ has the MAP. Hence, to verify that our sufficient conditions are non-trivial, we need to check whether our conditions implies conditions of Theorem 1. Fortunately, we can provide the negative answer to the above question. Indeed, we consider the dual of James tree space $J T^{*}$. As we know, $J T^{*}$ has the s-weak MAP with conjugate operators. On the other hand, $J T^{* *}$ and $J T^{*}$ have both the weak Radon-Nikodým property but $J T^{*}$ does not have the Radon-Nikodým property. Hence, our sufficient conditions are non-trivial.

We finally present the following interesting problems.

Problem 1. If $X^{*}$ has the s-weak MAP, then does $X^{*}$ has s-weak MAP with conjugate operators?

Problem 2. If $X^{*}$ has the AP and weak Radon-Nikodym property, then does $X^{*}$ has s-weak MAP?

If Problem 1 had an affirmative answer, then we would improve Theorem 4.4. In addition, if Problem 1 and Problem 2 had an affirmative answer, then we would improve Theorem 1 in Introduction.

\section{References}

[1] P. G. Cassaza, Approximate properties, In: W. B. Johnson, J. Lindenstrauss(eds.), Handbook of the Geometry of Banach Spaces, Volume 1, 271-316, Elsevier, 2001.

[2] C. Choi and J. M. Kim, Weak and quasi approximation properties in Banach spaces, J. Math. Anal. Appl. 316 (2006), no. 2, 722-735.

[3] — Hahn-Banach theorem for the compact convergence topology and applications to approximation properties, Houston J. Math. 37 (2011), no. 4, 1157-1164.

[4] T. Figiel and W. B. Johnson, The approximation property does not imply the bounded approximation property, Proc. Amer. Math. Soc. 41 (1973), 197-200. 
[5] A. Grothendieck, Produits tensoriels topologiques et espaces nucléires, Mem. Amer. Math. Soc. 16 (1955), no. 16, 140 pp.

[6] J. Johnson, Remarks on Banach spaces of compact operators, J. Funct. Anal. 32 (1979), no. $3,304-311$.

[7] K. Y. Lee, Dual spaces of compact operators space and the weak Radon-Nikodým property, Studia Math. 210 (2012), no. 3, 247-260.

[8] Å. Lima, O. Nygraard, and E. Oja, Isometric factorization of weakly compact operators and the approximation property, Israel J. Math. 119 (2000), 325-348.

[9] Å. Lima and E. Oja, The weak metric approximation property, Math. Ann. 333 (2005), no. $3,471-484$.

[10] J. Lindenstrauss and C. Stegall, Examples of separable spaces which do not contain $\ell_{1}$ and whose duals are non-separable, Studia Math. 54 (1975), no. 1, 81-105.

[11] K. Musial, The weak Radon-Nikodým property in Banach spaces, Studia Math. 64 (1978), no. 2, 151-174.

[12] E. Oja, The impact of the Radon-Nikodým property on the weak bounded approximation property, Rev. R. Acad. Cienc. Exactas Fis. Nat. Ser. A Math. 100 (2006), 325-331.

[13] R. A. Ryan, Introduction to Tensor Product of Banach Spaces, Springer, London, 2002.

Institute for Ubiquitous Information Technology and Applications

KONKUK UNIVERSITY

SEOUL 143-701, KoreA

E-mail address: northstar@kaist.ac.kr 